\title{
Imaging spectroscopy of quantum wells with interfacial fluctuations: A theoretical description
}

\author{
Omar Di Stefano, Salvatore Savasta, Giovanna Martino, and Raffaello Girlanda ${ }^{\text {a) }}$ \\ INFM and Dipartimento di Fisica della Materia e Tecnologie Fisiche Avanzate, Università di Messina, \\ Salita Sperone 31, I-98166 Messina, Italy
}

\begin{abstract}
We present a theoretical approach for the simulation of scanning local optical spectroscopy in disordered quantum wells (QWs). After a single realization of the disorder potential, we calculate spectra on a mesh of points on the QW plane, thus obtaining a three-dimensional matrix of data from which we construct two-dimensional spectroscopic images of excitons laterally localized at interface fluctuations. Our simulations are in close agreement with the experimental findings, and contribute to the interpretation of spatially resolved spectra in QWs.
\end{abstract}

[S0003-6951(00)04044-4]

Optoelectronics based on very thin active layers of semiconductor, such as quantum wells (QWs) are now currently used for many commercial applications. The performance of such devices is influenced by interface roughness, impurity concentration, and alloy homogeneity of the QW. Optical spectra of QWs yield valuable information on the quality of the interfaces and the growth process. ${ }^{1,2}$ Disorder in a $\mathrm{QW}$ results in an effective two dimensional (2D) potential spatially correlated which tend to localize the center of mass of the exciton. This image was put forth on the basis of detailed theoretical simulations ${ }^{3-5}$ and of observed homogeneous and inhomogeneous linewidths. ${ }^{1}$ Macroscopic optical probes ${ }^{1,2}$ have proven to be powerful techniques for probing localized excitons and hence interface fluctuations of quantum structures, however they perform a spatial averaging of the spectral signal, providing information at best on an inhomogeneous ensemble of quasi-0D (zero-dimensional) localized exciton states. Recently, techniques of very high spatial resolution optical spectroscopy allowed several groups to study the individual exciton eigenstates laterally localized at interface fluctuations. ${ }^{6-11}$ Stimulated by the relevance of the experimental results cited above, theoretical approaches modeling the interaction of quantum structures with highly inhomogeneous light fields have been recently presented. ${ }^{5,12-16}$

In this letter, we present theoretical results for scanning local optical spectroscopy in QWs obtained after the simulation of a disorder potential with prescribed statistical properties. We will consider an input light field with a given profile $g$ centered around the beam position $\mathbf{R}$. In experiments done by both illuminating and collecting with the same tip (reflection mode), the relevant optical signal that can be detected by a general near-field setup is proportional to ${ }^{17} S$ $=\int d z d \mathbf{r} \mathbf{P}^{*}(\mathbf{r}, z) \cdot \mathbf{E}(\mathbf{r}, z)$, where $\mathbf{P}(\mathbf{r}, z, \omega)$ is the macroscopic polarization of the sample induced by the electromagnetic field $\mathbf{E}(\mathbf{r}, z, \omega)$. We have indicated with $\mathbf{r} \equiv(x, y)$ the projection of the position vector on the plane of the QW and with $z$ the coordinate along the growth direction. Via interferometric techniques it is possible in principle to measure

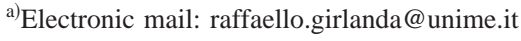

both the phase and the amplitude of $S$, thus obtaining information on the local complex susceptibility of a sample. ${ }^{18}$

Following the general linear response theory, the linear macroscopic polarization $\mathbf{P}(\mathbf{r}, z, \omega)$ can be written as ${ }^{4,14}$

$$
\mathbf{P}(\mathbf{r}, z, \omega)=\int \boldsymbol{\chi}\left(\mathbf{r}, z ; \mathbf{r}^{\prime}, z ; \omega\right) \mathbf{E}\left(\mathbf{r}^{\prime}, z^{\prime}, \omega\right) d \mathbf{r}^{\prime} d z^{\prime},
$$

where $\boldsymbol{\chi}\left(\mathbf{r}, z ; \mathbf{r}^{\prime}, z^{\prime} ; \omega\right)$ is the nonlocal susceptibility tensor. In systems with extended wave functions such as semiconductors nonlocality plays a relevant rule. ${ }^{14,16}$ In reasonable good quality QWs the amplitude of the confinement energy fluctuations are typically one order of magnitude smaller than the binding energy of the $1 S$ exciton. ${ }^{3}$ In this limit the relative exciton motion described by the effective wave function $\phi_{1 S}\left(\boldsymbol{\rho}_{e h}\right)$ may be assumed undistorted by disorder. Disorder affects significantly only the center of mass $(\mathrm{COM})$ motion through an effective potential $V(\mathbf{r})$. In particular, for semiconductor structures described within the usual envelope-function formalism with isotropic electron and hole dispersions and neglecting fine structure splittings, the susceptibility tensor becomes diagonal with identical elements given by $\mu_{v c}^{2} \phi_{1 S}(0)^{2} \rho(z) \rho\left(z^{\prime}\right) G\left(\mathbf{r}, \mathbf{r}^{\prime}, \omega\right)$, where $\mu_{v c}$ is the interband dipole moment, $\rho(z)$ is the product of the electron and hole envelope functions along the growth axis. The quantity $G\left(\mathbf{r}, \mathbf{r}^{\prime}, \omega\right)$ is the retarded propagator for the exciton COM motion. It obeys the following equation $(\hbar \omega+i \delta$ $\left.-\hat{H}_{\mathbf{r}}\right) G\left(\mathbf{r}, \mathbf{r}^{\prime}, \omega\right)=\delta\left(\mathbf{r}-\mathbf{r}^{\prime}\right)$. The Hamiltonian $\hat{H}_{\mathbf{r}}$, describing COM motion, is given by $\hat{H}_{\mathbf{r}}=-\left(\hbar^{2} / 2 M\right) \nabla^{2}+V(\mathbf{r})$ $+\hbar \omega_{0}$, where $\hbar \omega_{0}$ is the 1-S exciton energy level in the ideal disorder free QW. For the sake of simplicity in the following we describe the narrow light beam by a Gaussian EM-field profile propagating parallel to the QW growth direction $z, g(\mathbf{r})=\exp \left[-\left(x^{2}+y^{2}\right) / \sigma^{2}\right]$. The relevant optical field that can be detected by a general near-field setup as a function of the beam position and shape is thus proportional to

$$
S_{g}(\mathbf{R}, \omega)=\iint d \mathbf{r} d \mathbf{r}^{\prime} g(\mathbf{r}-\mathbf{R}) G\left(\mathbf{r}, \mathbf{r}^{\prime}, \omega\right) g\left(\mathbf{r}^{\prime}-\mathbf{R}\right) .
$$




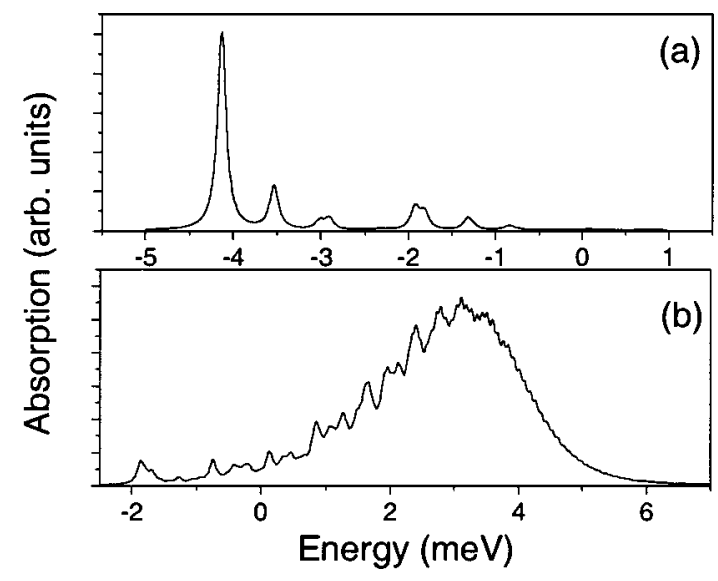

FIG. 1. (a) Local spectrum obtained centering the beam at the potential local minimum A indicated in Fig. 2(a); (b) local spectrum obtained centering the beam at the potential barrier B indicated in Fig. 2(a).

In the following we will present specific calculations for the imaginary part $S_{g}^{I}(\mathbf{R})$ of $S_{g}(\mathbf{R})$ which is proportional to the total absorption under local illumination. ${ }^{14-16}$ Although not direct, significant information on absorption under local illumination can also be obtained by subwavelength excitation spectroscopy. ${ }^{9,11}$ We model the random potential $V(\mathbf{r})$ felt by the excitons as a zero mean, Gauss distributed and spatially correlated process defined by the property $\left\langle V(\mathbf{r}) V\left(\mathbf{r}^{\prime}\right)\right\rangle=v_{0}^{2} e^{-\left|\mathbf{r}-\mathbf{r}^{\prime}\right|^{2} / 2 \xi^{2}}$, where $\langle\ldots\rangle$ denotes ensemble average over random configurations, $v_{0}$ is the width of the energy distribution, and $\xi$ is the correlation length characterizing the potential fluctuations. This widely adopted model $^{4}$ retains the main physical aspects in a very simple way.

The first step is the growth of the sample. Each sample corresponds to a single realization of disorder. Once $o b$ tained, the sample calculations are carried out in real space mapping on a fine mesh of points the Hamiltonian $\hat{H}_{\mathbf{r}}$, which is then tridiagonalized by using the Lanczos algorithm. ${ }^{15,16}$ We adopt an exciton kinetic mass of $m=0.25 m_{0}$ typical for AlAs/GaAs quantum wells. The local spectra have been calculated by considering a square region of $4 \mu \mathrm{m}^{2}$ which has been reproduced with a $500 \times 500$ mesh. Periodic boundary conditions have been adopted. For all the calculated spectra we used a homogeneous broadening $\delta=60 \mu \mathrm{eV}$. The calculations yield a three-dimensional matrix of data $S_{g}(x, y, \omega)$. Figure 1 shows two local spectra calculated with the beam position centered at two different positions and with a resolution $\sigma=20 \mathrm{~nm}$. Figure 1(a) has been obtained centering the beam at the potential local minimum A indicated in Fig. 2(a), the sharp peaks thus should origin from the OD exciton COM states localized inside the potential well. Figure 1(b) has been obtained centering the beam at the potential barrier B indicated in Fig. 2(a), the continuous band thus comes from quasi-2D states at higher energy. This picture will be further confirmed by the local optical images displayed below. Figure 2(a) displays a $2 \mathrm{D}$ gray scale image of the region of the specific realized sample where local spectra have been calculated. The labels A and B specify the locations chosen to calculate the local spectra in Fig. 1. The disordered potential has been obtained by using a correlation length $\xi=30 \mathrm{~nm}$ and a strength $v_{0}=1.5 \mathrm{meV}$. Figure 2(b) shows the global
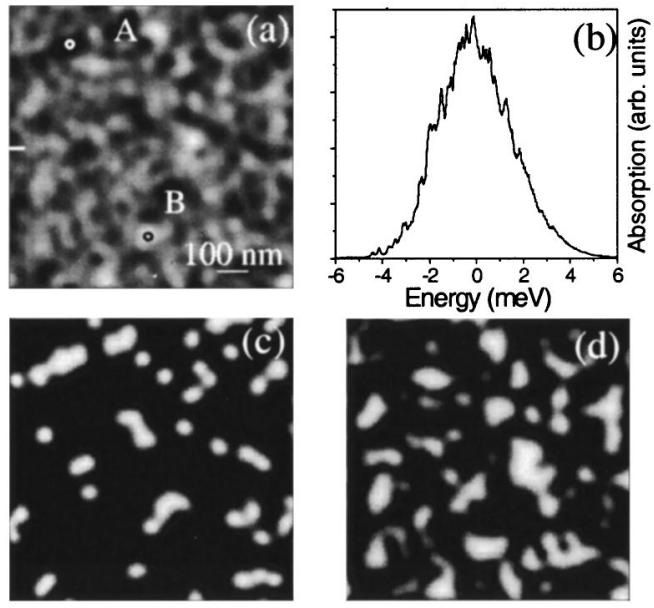

FIG. 2. (a) Specific realization of disorder potential in the region where local spectra have been calculated. The labels A and B specify the locations chosen to calculate the local spectra shown in Fig. 1; (b) global spectrum; (c) and (d) show the images obtained summing the local spectra, respectively, over the low and high energy tails of the global spectrum.

spectrum obtained replacing $g(\mathbf{r})$ with a constant. The $2 \mathrm{D}$ gray scale images shown in Figs. 2(c) and 2(d) have been obtained by summing $S_{g}^{I}(x, y, \omega)$ over spectral windows corresponding to the low energy (up to $-1.4 \mathrm{meV}$ ) and high energy tails (from $1.4 \mathrm{meV}$ ) of the global spectrum. We observe that the two images anticorrelate and are located, as expected, at the potential minima and at the barriers, respectively. These two complementary images give a precise information on the potential profile of the sample. Figure 3(i) displays a spectrum calculated with a beam with $\sigma$ $=250 \mathrm{~nm}$ and centered in the middle of the windows in Fig. 2(a). Despite the resolution not being too high, it is possible to observe a number of very sharp peaks. Figures $3(\mathrm{a})-3(\mathrm{~h})$ show five 2D images generated by sectioning the data $S_{g}^{I}(x, y, \omega)$ in planes of constant energy $\omega$ indicated by letters in Fig. 3(i). The images, calculated using a beam with $\sigma$ $=20 \mathrm{~nm}$, show the different behavior of two-dimensional ex-
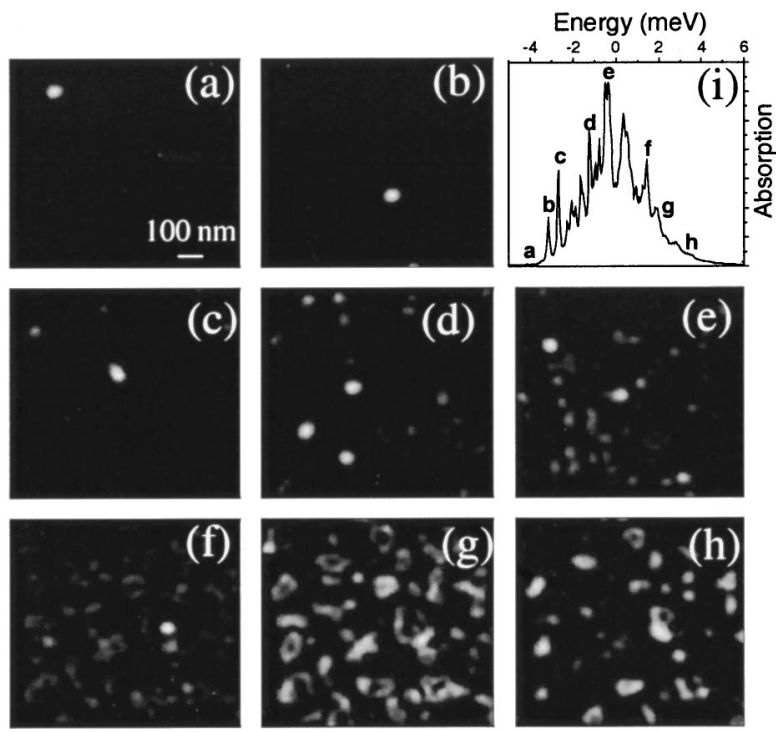

FIG. 3. (a)-(h) 2D images generated by sectioning the data $S_{g}^{I}(x, y, \omega)$ in planes of constant energy indicated by letters in (i); (i) spectrum obtained using a Gaussian beam with $\sigma=250 \mathrm{~nm}$ centered in the middle of the image in (a). 


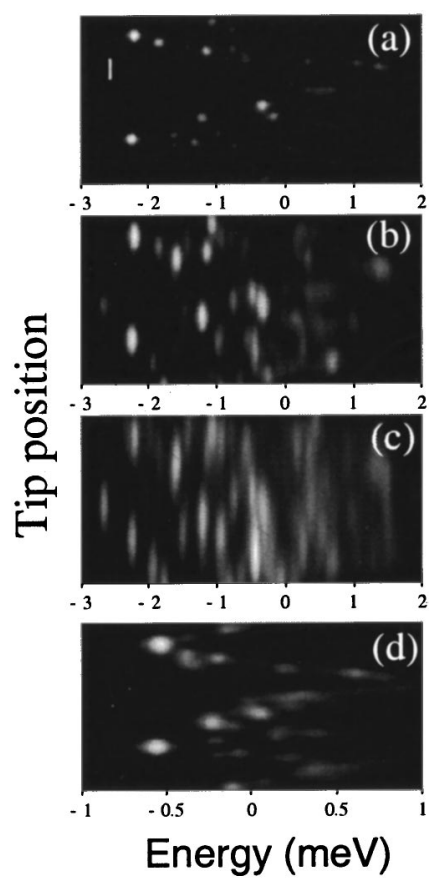

FIG. 4. (a) Local absorption as a function of beam position ( $x$ ) and photon energy obtained with a spatial resolution of $\sigma=20 \mathrm{~nm}$ (the white line is 0.1 $\mu \mathrm{m})$; (b) local absorption obtained with a spatial resolution of $\sigma=75 \mathrm{~nm}$; (c) local absorption obtained with a spatial resolution of $\sigma=150 \mathrm{~nm}$; (d) local absorption obtained with a spatial resolution of $\sigma=20 \mathrm{~nm}$ for the same potential of Fig. 2(a) reduced by a factor 3 .

citons when varying the excitation energy and give information on the spatial extension of the quantum states which determine the sharp peaks in Fig. 3(i). At low energy the images [Figs. 3(a)-3(e)] display the lowest states of the exciton center of mass motion located at the individual potential minima. Increasing the excitation energy, tunneling starts and we find an increasing number of structures which originate from excited states shared by different potential minima. An analogous behavior has been observed in experimental spectroscopic images. ${ }^{6,11}$ We also notice that the quite high spatial resolution chosen for the simulations $(\sigma=20 \mathrm{~nm})$ allows not only to look at the local absorption centers but also to partially determine their inner structure. Figure 4 shows the local absorption as a function of the beam position and energy $S_{g}^{I}\left(x, y_{0}, \omega\right)$ with $y=y_{0}$ fixed. The location of $y_{0}$ is indicated in Fig. 2(a). Figure 4(a), obtained with $\sigma$ $=20 \mathrm{~nm}$, clearly displays sharp structures from the individual eigenstates of the quantum systems which merge into quasi-continua near the potential barriers in agreement with the experimental findings. ${ }^{9}$ In order to investigate the impact that spatial resolution may have on the local spectra, we show in Figs. 4(b) and 4(c) the local spectra with the same potential used for Fig. 4(a) but with a lower spatial resolution (respectively, $\sigma=75 \mathrm{~nm}$ and $\sigma=150 \mathrm{~nm}$ ). We observe a broadening of the structures but also a modification of the relative intensities of the structures and the appearance of new peaks. This rather complex behavior is not merely an average effect but it also originates from spatial interference of the corresponding quantum states due to the nonlocal character of light-matter interaction in semiconductors according to Eq. (2). Figure 4(d) has been obtained reducing by a factor of three the strength of the disordered potential. We observe a reduction of the spectral region in agreement to what is generally observed in global spectra. We also observe a reduction of the excited states and an overall broadening of the structures.

In summary we have presented a theoretical formulation of scanning local optical spectroscopy in disordered quantum wells. The two-dimensional images presented here show that our theoretical simulations can give a useful contribution to the interpretation of experimental investigations and characterizations. Furthermore, the direct comparison of spectroscopic images with the realized disorder potential here shown, puts forward the correlation between structural disorder and spectroscopic near-field images in QWs. We also expect that the approach here presented can be applied to other mesoscopic photosensitive two-dimensional systems.

The authors acknowledge the support of INFM through the Project NanoSNOM.

${ }^{1}$ C. Weisbuch, R. Dingle, A. C. Grossard, and W. Wiegmann, Solid State Commun. 37, 219 (1981).

${ }^{2}$ M. A. Herman, D. Bimberg, and J. Christen, J. Appl. Phys. 70, R1 (1991).

${ }^{3}$ R. F. Schnabel, R. Zimmermann, D. Bimberg, H. Nickel, R. Lösch, and W. Schlapp, Phys. Rev. B 46, 9873 (1992).

${ }^{4}$ S. Glutsch, D. S. Chemla, and F. Bechstedt, Phys. Rev. B 54, 11592 (1996).

${ }^{5}$ E. Runge and R. Zimmermann, Phys. Status Solidi B 206, 167 (1998).

${ }^{6}$ H. F. Hess, E. Betzig, T. D. Harris, L. N. Pfeiffer, and K. W. West, Science 264, 1740 (1994).

${ }^{7}$ D. Gammon, E. S. Snow, and D. S. Katzer, Appl. Phys. Lett. 67, 2391 (1995).

${ }^{8}$ D. Gammon, E. S. Snow, B. V. Shanabrook, D. S. Katzer, and D. Park, Science 273, 87 (1996).

${ }^{9}$ D. Gammon, E. S. Snow, B. V. Shanabrook, D. S. Katzer, and D. Park, Phys. Rev. Lett. 76, 3005 (1996).

${ }^{10}$ R. Cingolani, F. Sogawa, Y. Arakawa, L. Vanzetti, L. Sorba, and A. Franciosi, Appl. Phys. Lett. 73, 148 (1998).

${ }^{11}$ Q. Wu, R. D. Grober, D. Gammon, and D. S. Katzer, Phys. Rev. Lett. 83, 2652 (1999).

${ }^{12}$ B. Hanewinkel, A. Knorr, P. Thomas, and S. W. Koch, Phys. Rev. B 55, 13715 (1997).

${ }^{13}$ G. W. Bryant, Appl. Phys. Lett. 72, 768 (1998).

${ }^{14}$ O. Mauritz, G. Goldoni, F. Rossi, and E. Molinari, Phys. Rev. Lett. 82, 847 (1999).

${ }^{15}$ S. Savasta, G. Martino, and R. Girlanda, Phys. Rev. B 61, 13852 (2000).

${ }^{16}$ O. Di Stefano, S. Savasta, G. Martino, and R. Girlanda, Phys. Rev. B (to be published).

${ }^{17}$ J.-J. Greffet and R. Carminati, Prog. Surf. Sci. 56, 133 (1997).

${ }^{18}$ F. Zenhausern, Y. Martin, and H. K. Wickramasinghe, Science 269, 1083 (1995). 